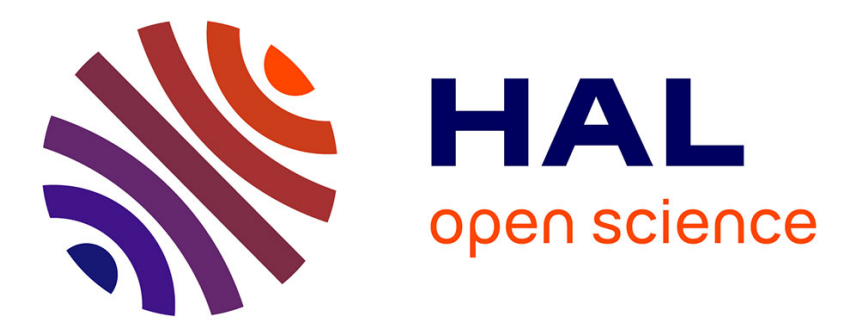

\title{
Global structure and sodium ion dynamics in Mercury's magnetosphere with the offset dipole
}

Manabu Yagi, Kanako Seki, Y. Matsumoto, Dominique C. Delcourt, François Leblanc

\section{- To cite this version:}

Manabu Yagi, Kanako Seki, Y. Matsumoto, Dominique C. Delcourt, François Leblanc. Global structure and sodium ion dynamics in Mercury's magnetosphere with the offset dipole. Journal of Geophysical Research Space Physics, 2017, 122 (11), pp.10,990-11,002. 10.1002/2017JA024082 . insu01585106

\section{HAL Id: insu-01585106 \\ https://hal-insu.archives-ouvertes.fr/insu-01585106}

Submitted on 7 May 2020

HAL is a multi-disciplinary open access archive for the deposit and dissemination of scientific research documents, whether they are published or not. The documents may come from teaching and research institutions in France or abroad, or from public or private research centers.
L'archive ouverte pluridisciplinaire HAL, est destinée au dépôt et à la diffusion de documents scientifiques de niveau recherche, publiés ou non, émanant des établissements d'enseignement et de recherche français ou étrangers, des laboratoires publics ou privés. 


\section{Journal of Geophysical Research: Space Physics}

\section{RESEARCH ARTICLE \\ 10.1002/2017JA024082 \\ Global Structure and Sodium Ion Dynamics in Mercury's Magnetosphere With the Offset Dipole}

Key Points:

- North-south asymmetry is of paramount importance in the global structure of Mercury's magnetosphere

- Southward precipitation is dominant for planetary sodium ions

- High energy "sodium ring" can be formed under nominal solar wind conditions at Mercury's orbit

Correspondence to: M. Yagi,

manabu.yagi@riken.jp

\section{Citation:}

Yagi, M., Seki, K., Matsumoto, Y., Delcourt, D. C., \& Leblanc, F. (2017). Global structure and sodium ion dynamics in Mercury's magnetosphere with the offset dipole. Journal of Geophysical Research: Space Physics, 122 10,990-11,002. https://doi.org/10.1002/ 2017JA024082

Received 23 FEB 2017

Accepted 1 SEP 2017

Accepted article online 5 SEP 2017

Published online 3 NOV 2017

(C2017. American Geophysical Union. All Rights Reserved.

\author{
M. Yagi ${ }^{1}$, K. Seki ${ }^{2}$ iD, Y. Matsumoto ${ }^{3}$ D, D. C. Delcourt ${ }^{4}$ iD, and F. Leblanc ${ }^{5}$ iD \\ ${ }^{1}$ Advanced Institute for Computational Science, RIKEN, Kobe, Japan, ${ }^{2}$ Department of Earth and Planetary Science, Graduate \\ School of Science, University of Tokyo, Bunkyo, Japan, ${ }^{3}$ Graduate School of Science, Chiba University, Chiba, Japan, ${ }^{4}$ LPP, \\ Ecole Polytechnique, CNRS, Paris, France, ${ }^{5}$ LATMOS, IPSL, CNRS, Paris, France
}

Abstract We conducted global magnetohydrodynamics (MHD) simulation of Mercury's magnetosphere with the dipole offset, which was revealed by MESSENGER (Mercury Surface, Space Environment, Geochemistry, and Ranging) observations, in order to investigate its global structure under northward interplanetary magnetic field conditions. Sodium ion dynamics originating from the Mercury's exosphere is also investigated based on statistical trajectory tracing in the electric and magnetic fields obtained from the MHD simulations. The results reveal a north-south asymmetry characterized by open field lines around the southern polar region and northward deflection of the plasma sheet in the far tail. The asymmetry of magnetic field structure near the planet drastically affects trajectories of sodium ion and thus their pressure distributions and precipitation pattern onto the planet. Weaker magnetic field strength in the southern hemisphere than in the north increases ion loss by precipitation onto the planetary surface in the southern hemisphere. The "sodium ring," which is formed by high-energy sodium ions drifting around the planet, is also found in the vicinity of the planet. The sodium ring is almost circular under nominal solar wind conditions. The ring becomes partial under high solar wind density, because dayside magnetosphere is so compressed that there is no space for the sodium ions to drift around. In both cases, the sodium ring is formed by sodium ions that are picked up, accelerated in the magnetosheath just outside the magnetopause, and reentered into the magnetosphere due to combined effects of finite Larmor radius and convection electric field in the dawnside magnetosphere.

\section{Introduction}

In situ observation by Mercury Surface, Space Environment, Geochemistry, and Ranging (MESSENGER) from 2011 to 2015 discovered new aspects of Mercury's magnetosphere. Previous observation by Mariner 10 discovered Mercury's magnetosphere, and the intrinsic magnetic field of Mercury was estimated to be 300-400 nT at magnetic equator (Ness et al., 1975). Statistical analysis of orbital observations by MESSENGER suggested the strength of intrinsic magnetic field of Mercury to be $195 \mathrm{nT}$ (Anderson et al., 2011), which is about two thirds of the previous estimation. The dipole field is much weaker than that of the Earth's, that is, 1/150 compared at planetary surface and 1/2800 for the magnetic moment. However, the intrinsic magnetic field still has enough strength to sustain a magnetosphere and to prevent direct interaction between the solar wind and planetary surface under nominal conditions.

One of the important results from MESSENGER from the magnetospheric point of view is the discovery of a "dipole offset" (Anderson et al., 2011), which was originally suggested by Mariner 10 flyby observations (Ng \& Beard, 1979; Whang, 1977) but with a large uncertainty. The magnetic equator is thought to be shifted northward by $485 \mathrm{~km}$, which is equivalent to $0.2 R_{M}$, where $R_{M}$ is the radius of Mercury ( $\left.2440 \mathrm{~km}\right)$. Observations also show dynamic activities of Mercury's magnetosphere such as "substorm"-like events associated with magnetic reconnection and electron acceleration (Slavin et al., 2012), or suprathermal electron burst events (Ho et al., 2011) also revealed by Mariner 10 (Simpson et al., 1974). Dawn-dusk asymmetry on ion composition (Raines et al., 2013) and high plasma pressure near Mercury's surface (Korth et al., 2012) are two other characteristics of Mercury's magnetosphere discovered by MESSENGER.

Despite the numerous achievements of MESSENGER, characteristics of the magnetosphere remains ambiguous when observed from a single point of observation. Therefore, theoretical approaches such as simulation and modeling have been used to understand Mercury's magnetosphere. Development of computational resources in addition to advancement of calculation scheme have enabled us to perform 
three-dimensional global simulations of planetary magnetosphere with enough resolution to properly describe Mercury's magnetosphere. A number of MHD simulations have revealed the characteristics of Mercury's magnetosphere such as bow shock, magnetopause, plasma sheet, and magnetospheric cusp structures (Benna et al., 2009; Ip \& Kopp, 2002; Jia et al., 2015; Kabin et al., 2000, 2008; Kidder et al., 2008; Pantellini et al., 2015; Varela et al., 2015; Yagi et al., 2010). One of the important characteristics of Mercury's magnetosphere is a strong north-south asymmetry because of the strong $B_{x}$ component of the interplanetary magnetic field (IMF). The global configuration can be described by MHD theory; however, kinetic effects of nonthermal ions might violate the MHD approach in some cases, in small system like Mercury's magnetosphere. Hybrid models of the interaction of the solar wind protons with a dipole magnetic field show some characteristics such as dawn-dusk asymmetry even though there is no IMF- $B_{y}$ component (Kallio \& Janhunen, 2003), and the formation of a hot plasma ring (Hercik et al., 2016; Travnicek et al., 2007) which were not produced by MHD simulation. Kinetic effects become even more important for heavier ions, which originate from Mercury's exosphere (sodium, potassium, and calcium). Trajectory tracings of test particles show that the centrifugal acceleration due to curvature of the $\mathbf{E} \times \mathbf{B}$ drift path is sufficiently efficient to transport the ions from high latitudes to low latitudes (Delcourt et al., 2003, 2012, 2007).

Yagi et al. (2010) showed the formation of high-energy "sodium ring" based on statistical trajectory tracings of sodium ions originating from the exosphere using $\mathbf{E}$ and $\mathbf{B}$ fields derived from MHD simulations under northward IMF conditions. Sodium ions originating from the exosphere undergo acceleration either in the magnetosheath or in the magnetosphere up to energies where quasi-trapped motion due to magnetic drift in the intrinsic dipolar magnetic field is dominant and can form a high-pressure region around the planet. The ring-shaped or partial ring-shaped high-pressure region is called the sodium ring. The acceleration mechanisms of sodium ions as well as the shape of the ring are dependent of the solar wind conditions. Because of the large Larmor radius, formation of ring-shaped sodium ring is more sensitive to the solar wind conditions than proton ring shown by Travnicek et al. $(2007,2010)$.

Naturally, most of the simulation studies before MESSENGER's era use the intrinsic magnetic field model derived from Mariner 10 and thus overestimated the intrinsic magnetic field strength of Mercury and did not introduce an offset of the dipole. Therefore, one of the important questions discussed in this paper is to know how a weaker and offset dipole intrinsic magnetic field would alter Mercury's magnetosphere. In this study, we investigate how this new intrinsic magnetic field model can impact the sodium ring and its formation mechanism. In section 2, we first show the results of MHD simulations using the weaker and offset dipole intrinsic magnetic field based on the MESSENGER's observation. Next, trajectory tracings of test particles of sodium ions in the $\mathbf{E}$ and $\mathbf{B}$ fields obtained from the MHD simulation results are shown in section 3 . In section 4, we discuss the changes and similarities of the magnetospheric structure obtained with this new internal magnetic field model. The conclusions for the sodium ion dynamics and the formation of the sodium ring are summarized in section 5 .

\section{MHD Simulation of Mercury's Magnetosphere With Offset Dipole}

\subsection{Basic Equation}

In order to simulate the global structure of Mercury's magnetosphere, we use the following ideal magnetohydrodynamics (MHD) approximation. Normalized equations as well as numerical schemes are similar to those in Yagi et al. (2010),

$$
\begin{gathered}
\frac{\partial \rho}{\partial t}=-(\boldsymbol{v} \cdot \nabla) \rho-\rho(\nabla \cdot \boldsymbol{v}) \\
\frac{\partial v}{\partial t}=-(\boldsymbol{v} \cdot \nabla) \mathbf{v}-\frac{1}{2 \rho} \nabla P-\frac{1}{\rho} \mathbf{J} \times \mathbf{B} \\
\frac{\partial P}{\partial t}=-(\boldsymbol{v} \cdot \nabla) P-\gamma P(\nabla \cdot \mathbf{v})
\end{gathered}
$$




$$
\frac{\partial \mathbf{A}}{\partial t}=-\mathbf{E}-\nabla \boldsymbol{\phi}
$$

where $\rho, \mathbf{v}, P$, and $\mathbf{A}$ indicate density, velocity, pressure, and vector potential. Magnetic field $\mathbf{B}$, current density $\mathbf{J}$, and electric field $\mathbf{E}$ are defined as follows:

$$
\begin{gathered}
\mathbf{B}=\mathbf{B}_{0}+\nabla \times \mathbf{A} \\
\left.\mathbf{J}=\nabla \times \mathbf{B}=\nabla(\nabla \cdot \mathbf{A})-\nabla^{2} \mathbf{A}\right) \\
\mathbf{E}=-\mathbf{v} \times \mathbf{B}=(\mathbf{v} \cdot \nabla) \mathbf{A}-(\nabla \mathbf{A}) \cdot \mathbf{V}
\end{gathered}
$$

Vector potential $\mathbf{A}$ is introduced to solve the equation instead of the magnetic field $\mathbf{B}$ to limit numerical error on $\nabla \cdot \mathbf{B}$, which would significantly violate the adiabatic invariant. $\mathbf{B}_{0}$ is the background magnetic field which is separated from the variation $\mathbf{B}$ to reduce the error when calculating the current density $\mathbf{J}$. In this study, $\mathbf{B}_{0}$ is the intrinsic magnetic field of Mercury, defined as

$$
\begin{gathered}
B_{0 x}=-\frac{M_{H}}{r^{5}} 3 x z^{\prime} \\
B_{0 y}=-\frac{M_{H}}{r^{5}} 3 y z^{\prime} \\
B_{0 z}=-\frac{M_{H}}{r^{5}}\left(3 z^{\prime 2}-r^{\prime 2}\right)
\end{gathered}
$$

Where $r^{\prime}$ is the distance from the dipole center, and $z^{\prime}$ is its projection on the dipole axis, which includes the offset from the planetary center by $485 \mathrm{~km}$, and $M_{H}$ is the dipole moment equivalent to $195 \mathrm{nT}$ at magnetic equator. We adopted the R-CIP algorithm (Xiao et al., 1996; Yabe \& Aoki, 1991) to solve the advection equation. The nonadvection terms are solved by Runge-Kutta method in time and using a fourth-order central difference for space discretization. One of the issues on this algorithm is to solve a strong velocity divergence, especially shock capturing so that we introduced artificial viscosity effective only at the shock (Ogata \& Yabe, 1999). Boundary conditions at Mercury are one of the uncertain issues of modeling magnetospheres. The global configuration, such as the location of the plasma sheet boundary as well as magnetospheric convection, depends largely on the conductivity inside Mercury (Janhunen \& Kallio, 2004; Jia et al., 2015; Seki et al., 2013) especially in the south IMF condition. In this study, the boundary is set to be 'reflective," that is, no flux penetrates the planetary surface and no pressure gradient is introduced along the radial direction at the surface. In order to compare with previous study of the sodium ring, we adopted the same boundary conditions as those used in Yagi et al. (2010).

\subsection{Global MHD Model of Mercury's Magnetosphere}

The MHD model is essentially the same as in Yagi et al. (2010), the principal change being the update of the intrinsic magnetic field model based on MESSENGER's observations, i.e., $195 \mathrm{nT} /\left(R_{M}{ }^{3}\right)$ at the magnetic equator and a dipole offset of $0.2 R_{M}$ northward (Anderson et al., 2011). The IMF is northward, $B=[0,0,10] \mathrm{nT}$, while typical IMF at Mercury's orbit contains strong $B_{x}$ which would cause north-south asymmetry of the magnetosphere (Kabin et al., 2000). The main interest of this study is how the dipole offset affects the global configuration of Mercury's magnetosphere especially its north-south asymmetry so that we chose to neglect the IMF $B_{x} / B_{y}$ components.

\subsubsection{Case 1: Typical Solar Wind Case}

In Case 1, solar wind parameters are given as $\rho=35 \mathrm{~cm}^{-3}$ and $v_{x}=400 \mathrm{~km} / \mathrm{s}$, corresponding to typical values at the Mercury's aphelion. The solar wind parameters are identical to those used in Case 1 of Yagi et al. (2010). 
(a)

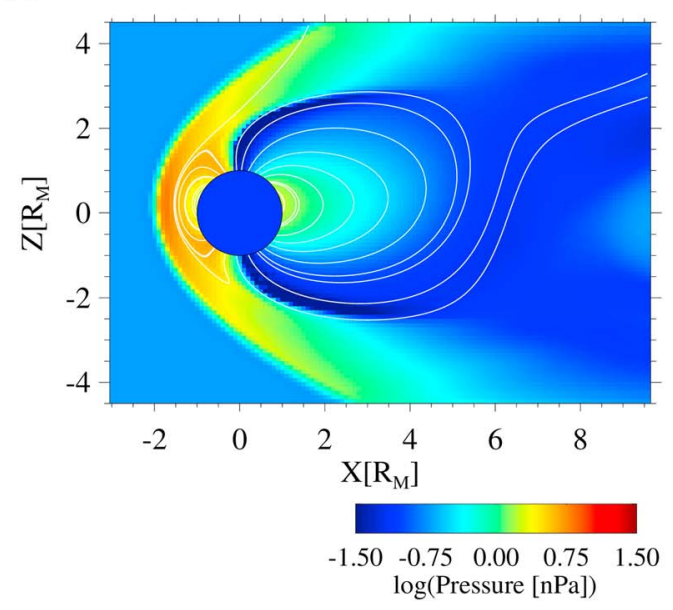

(c)

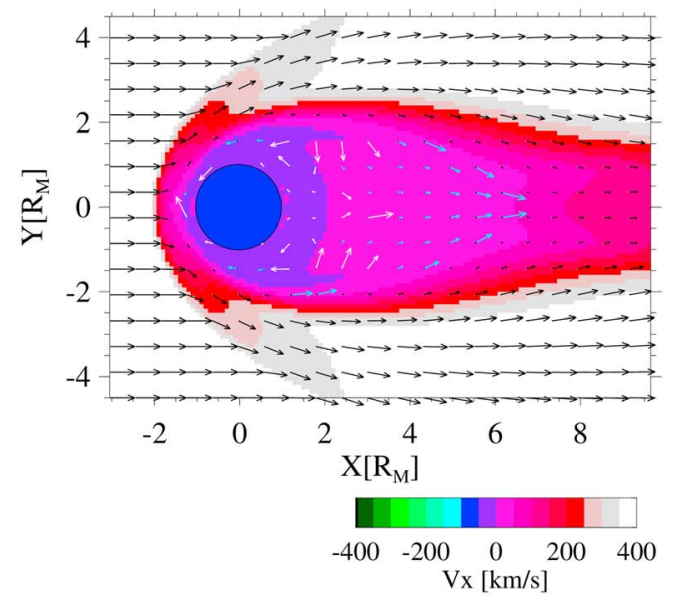

(b)

Case 2: High SW density

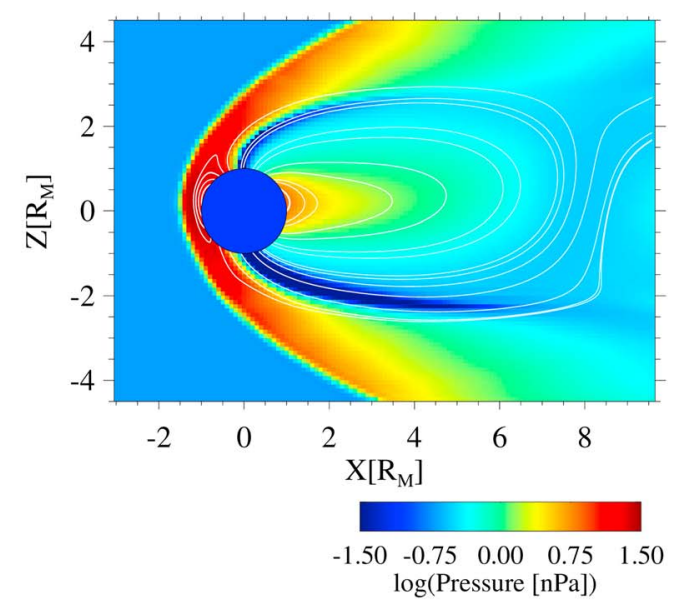

(d)

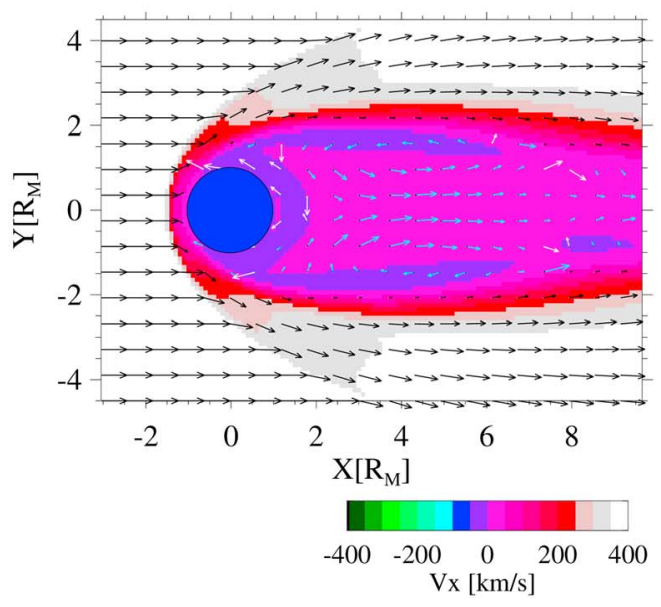

Figure 1. Global MHD simulation results of Mercury's magnetosphere. (a, b) Pressure in logarithmic scale (color scale) and magnetic field lines (white lines) in meridian plane. $(c, d) v_{X}$ in color and velocity vector with arrows in $z=0.2 R_{M}$ plane, which corresponds to the magnetic equator of Mercury's internal field. To emphasize the slow velocity pattern in the magnetosphere, sky blue arrows are added where velocity is less than $40 \mathrm{~km} / \mathrm{s}$ and white arrows for less than $5 \mathrm{~km} / \mathrm{s}$, and blue contour indicates the return flow region. Figures $1 \mathrm{a}$ and $1 \mathrm{c}$ show the results of Case 1 (typical solar wind density case: $\rho=35 \mathrm{~cm}^{-3}$ ) and Figures $1 \mathrm{~b}$ and $1 \mathrm{~d}$ show the results of Case 2 (high solar wind density case: $\rho=140 \mathrm{~cm}^{-3}$ ).

Figure 1 shows the quasi-steady state of the MHD simulation for Case 1. The subsolar magnetopause (MP) and bow shock (BS) are located at $1.4 R_{M}$ and $1.7 R_{M}$, respectively. Figure $2 \mathrm{a}$ shows the pressure distribution at the planetary surface. "Cusp" structures are clearly seen from 50 to $80^{\circ}$ of latitude in the northern hemisphere and from -70 to $-30^{\circ}$ in the southern hemisphere. The southern cusp region is larger than the northern cusp because the intrinsic magnetic field is weaker in the southern hemisphere due to the dipole offset. The results indicate that the dayside magnetosphere is strongly compressed. However, fundamental structures such as the bow shock, magnetopause, and cusp are unchanged.

One of the outstanding characteristics is an open field line region around the south pole. This open field line region indicates that the magnetosphere becomes asymmetric not only with respect to the geographic equatorial plane but also with respect to the magnetic equator. This characteristic is not shown in the previous model, which has no dipole offset, so it is caused by the offset of dipole. The global convection pattern in the $X-Y$ plane is almost tailward, while a weak return flow from tailward to dayside at the vicinity of Mercury is also seen (Figure 1c).

\subsubsection{Case2: High Solar Wind Dynamic Pressure Case}

In Case 2, solar wind parameters are set to $\rho=140 \mathrm{~cm}^{-3}$, and $v_{x}=400 \mathrm{~km} / \mathrm{s}$. The dynamic pressure is 4 times higher than that in Case 1 and corresponds to Case 2 in Yagi et al. (2010). In Case 2, the bow shock at the 
(a)Case 1: Nominal SW density

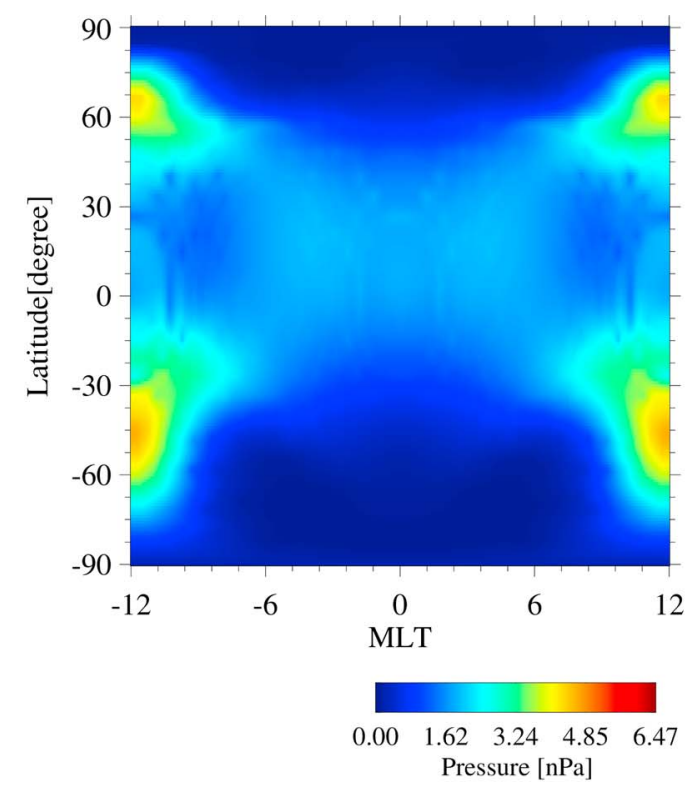

(b)Case 2: High SW density

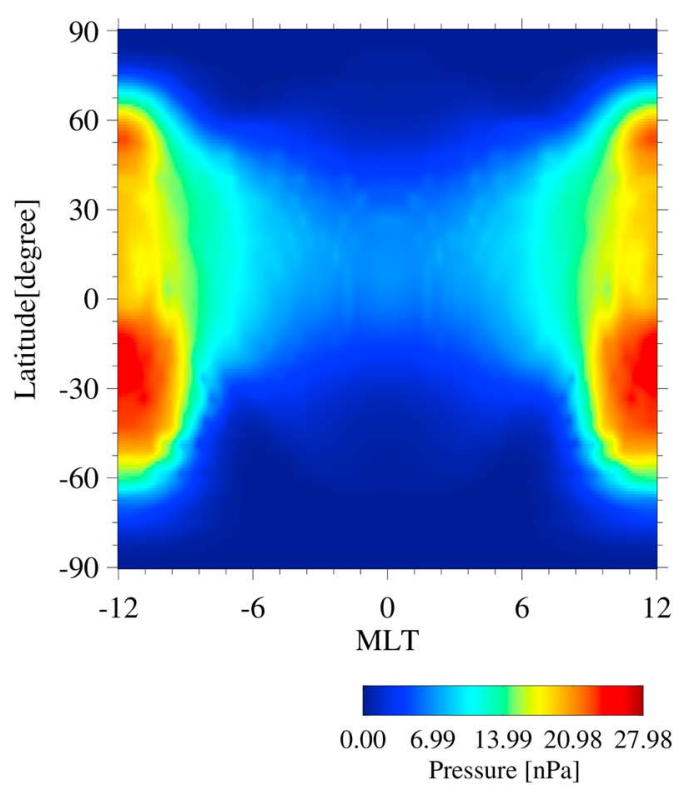

Figure 2. Pressure distributions at the planetary surface obtained from the MHD simulations. (a) Typical solar wind conditions and (b) high solar wind dynamic pressure case.

subsolar point is located at $1.5 R_{M}$, while the magnetosphere is so compressed that it is difficult to clearly identify the magnetopause. Open-closed boundary of the magnetic field lines, one of the definitions used for the magnetopause, is located at $1.2 R_{M}$. However, the intrinsic magnetic field cannot sustain the magnetosphere from the solar wind so that the pressure does not decrease inside the "magnetopause" on the dayside. As shown in Figure $2 b$, the cusp regions are also difficult to define because the high-pressure region from the southern hemisphere expands from $-60^{\circ}$ latitude up to the equator. In the northern hemisphere, the cusp region can be identified from 40 to $60^{\circ}$ latitude, while the pressure from 0 to $40^{\circ}$ areas also significantly increases. Thus, it is difficult to clearly separate the northern and southern cusps. For these reasons, the magnetopause cannot be clearly identified especially in the southern hemisphere. In the nightside, the dipole field is more stretched tailward than in Case 1. The global convection pattern in Case 2 is also analogous to that in Case 1; that is, the tailward flow is dominant in the most part, while the weak return flow exists at the vicinity of the planet.

\section{Distribution of $\mathrm{Na}+$ lons in Mercury's Magnetosphere}

\subsection{Numerical Model}

In small-scale plasma circulation system as Mercury's magnetosphere, Larmor radii of heavy ions become comparable to the magnetic field structures or even system size. Mercury has no thick atmosphere; however, the existence of a sodium exosphere is known by ground-based optical observations since Potter and Morgan (1985), heavy ions being supplied from the exosphere into the magnetosphere by photoionization. In this study, we performed statistical trajectory tracing of sodium ions in the electric and magnetic field calculated by MHD simulation shown in the previous section. The initial state of sodium ions is based on the sodium exosphere model given by Leblanc et al. (2003), which is the same model as that used in our previous paper, and over 270,000 test particles are solved in this study (see Figure 4 in Yagi et al., 2010). Assuming that Mercury's magnetosphere is in steady state, each momentum value of density, average velocity, and pressure can be defined as follows:

$$
\rho=\frac{\sum\left(F_{i} t_{i}\right)}{\Delta x^{3}}
$$




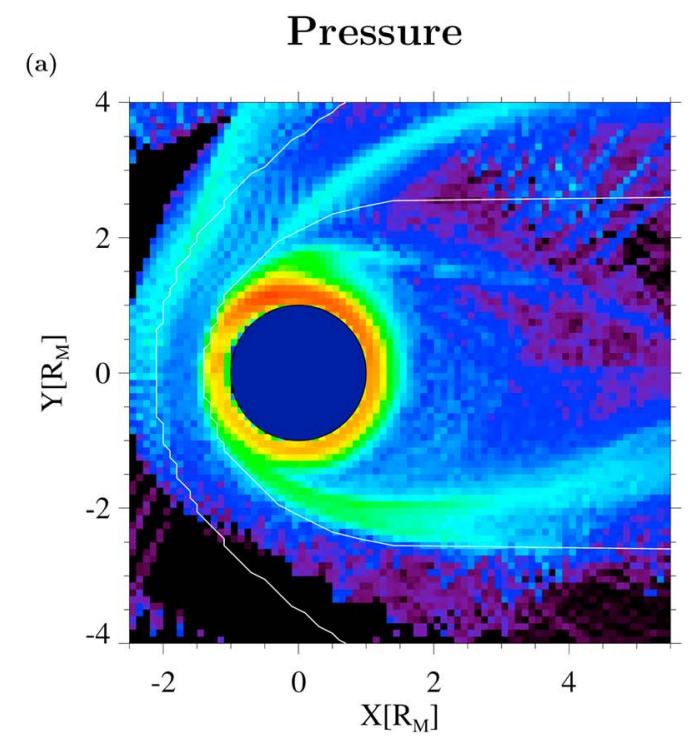

(c)

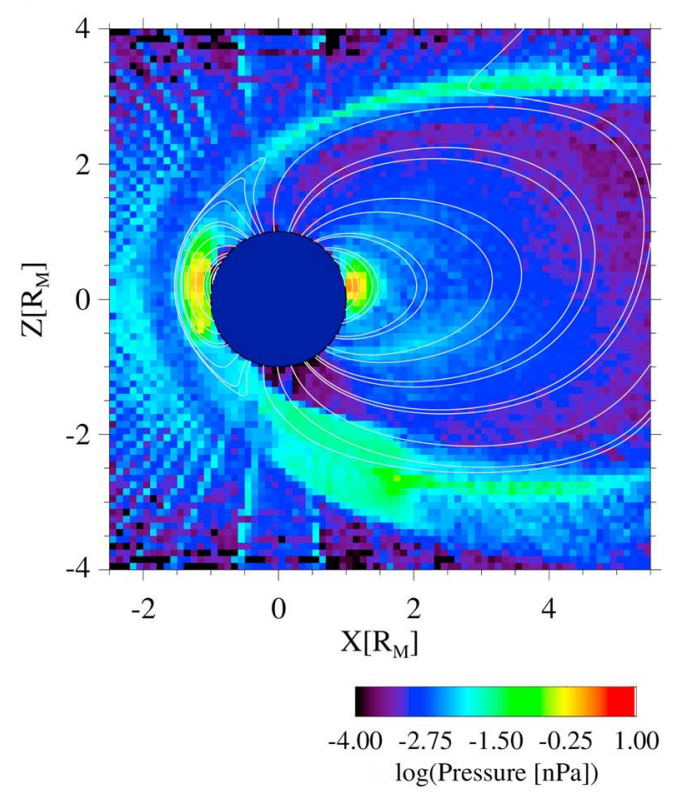

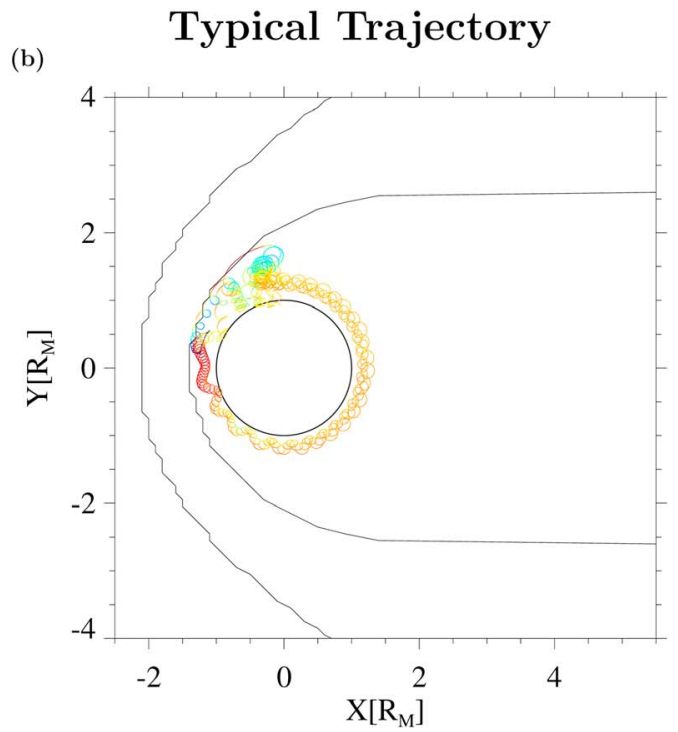

(d)

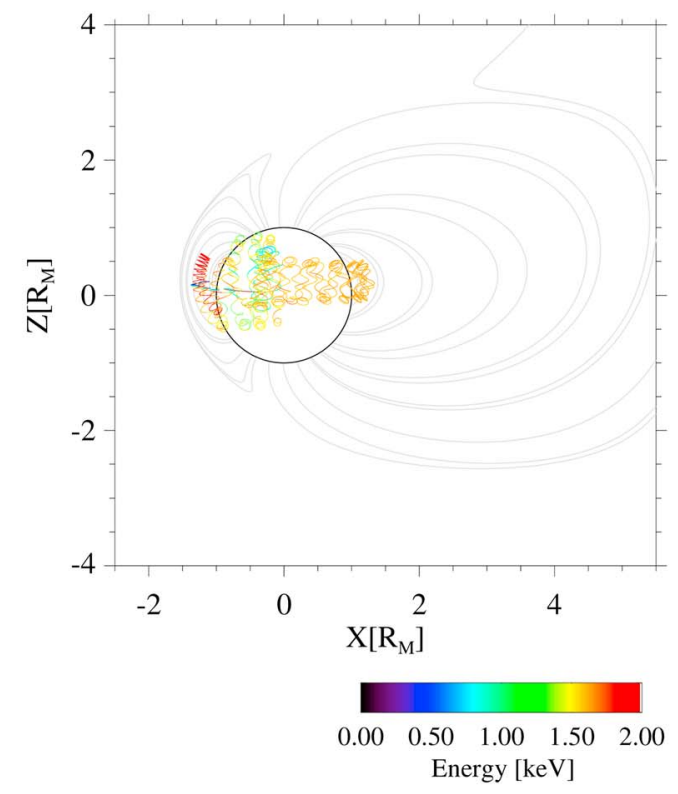

Figure 3. $(a, c)$ The pressure distribution of sodium ions (color) obtained from statistical trajectory tracing together with the field lines (white lines) for the nominal solar wind case (Case 1). (b, d) A sample trajectory of sodium ions that form the "sodium ring," i.e., the high-pressure region around the planet. The color of the trajectory line indicates the energy of the test sodium ion. Figures $3 \mathrm{a}$ and $3 \mathrm{~b}$ show the $z=0.2 R_{M}$ plane (magnetic equatorial plane), and Figures $3 \mathrm{c}$ and $3 \mathrm{~d}$ correspond to the meridian plane.

$$
\begin{gathered}
\overline{\mathrm{V}}=\frac{\sum\left(F_{i} t_{i} V_{i}\right)}{\rho \Delta x^{3}} \\
P=\frac{\sum\left(F_{i} t_{i}\left(V_{i}-\bar{V}\right)^{2}\right)}{\rho \Delta x^{3}}
\end{gathered}
$$

where subscript $i$ indicates the index of test particle. $F_{i}, t_{i}, V_{i}$ and $\Delta x^{3}$ are the weight of the test particle per unit time, the resident time of a test particle in each cell, the velocity of the particle in the cell, and the cell volume, respectively. 


\subsection{Case 1: Typical Solar Wind Case}

Figures $3 a$ and $3 c$ show the pressure distribution of sodium ions for Case 1, obtained from statistical trajectory tracing. In the magnetic equatorial plane, high-energy sodium ions are found around the planet. This sodium ring consists of several keV sodium ions drifting around the planet. A typical trajectory of sodium ions in the sodium ring is shown in Figures $3 \mathrm{~b}$ and $3 \mathrm{~d}$. This sodium ion is picked up in the magnetosphere in $z=0.2 R_{M}$ plane (magnetic equatorial plane of the intrinsic dipole field) and drifted because of the convection electric field toward the dayside magnetopause. Upon encountering the dayside magnetopause, the ion is accelerated to several keV by the electric field in the magnetosheath. Because of the large Larmor radius, the ion meanders between the magnetosphere and magnetosheath while drifting along the magnetopause. Notice that most ions will drift along the magnetopause and escape from the magnetosphere, but only a part of ions can reenter in the magnetosphere across the magnetopause by the cancelation between the convection and the magnetic drift along the $x$ direction as discussed in Yagi et al. (2010). Upon entering deep enough into the magnetosphere, the energetic ion drifts around the planet, thanks to the magnetic gradient of the dipole-dominated magnetic field. For nominal solar wind conditions used in Case 1, the dayside magnetosphere is compressed, but there are still enough space for sodium ions to drift around the dayside magnetosphere.

Notice that stripe shapes are found in the solar wind region ( $X-Z$ plane) as well as tailward magnetosheath in the dawnside $(X-Y)$. These structures come from initial locations of test particles and indicates the statistical errors. The particle counts in each bin of these regions are less than 10, while over 1,000 counts are found around the sodium ring, and over several 100 counts are found in the lobe regions, so it is feasible to discuss the momentum values in the magnetosphere.

Figures $5 \mathrm{a}$ and $5 \mathrm{c}$ show the energy and energy flux of sodium ions precipitating onto the planetary surface in Case 1 . On the nightside, a precipitation band of several $\mathrm{keV}$ is the most obvious feature in Figures $5 \mathrm{a}$ and $5 \mathrm{c}$ at midnight near equator. This band originates from precipitating sodium ring atoms with large Larmor radius and extends from 6 MLT toward dayside via nightside. Narrow precipitation bands at high latitude of several tens to hundreds of $\mathrm{eV}$ at 60 , and -30 to $-60^{\circ}$ latitude are also found. This energy range is too low for pick up energy induced by the magnetosheath convection, thus is not related to sodium ring. Instead, this energy range is associated with magnetospheric convection electric field. These bands consist of ions picked up at high latitudes at the boundary between "return flow region" that stands at the vicinity of the planet, and "outflow region" that stands outside of return flow region. The structure of the whole magnetosphere is almost ellipsoid, and these two regions are characterized by convection patterns (as shown in Figure 1c). lons picked up in the return flow-dominant region will first drift toward dayside magnetopause. Part of these ions will be the sodium ring. On the other side, ions picked up in the outflow region contribute to a tailward escape channel. Low-energy ions in this boundary region are almost at rest. Still, some of them may get energized via gradient drift along the convection electric field and may precipitate after flowing along the magnetic field lines. There are also precipitation in the cusps indicated by the magenta contour lines (MHD pressure countour map displayed in Figure 2). Precipitation occurs in a wider region around the southern cusp that is wider than in the northern cusp. There are also precipitations of several eV ions in various places at the surface which are ions precipitating immediately after ionization.

\subsection{Case 2: High Solar Wind Dynamic Pressure Case}

Figures 4a and 4c show the distribution of sodium ions in Mercury's magnetosphere obtained from the statistical tracing. In this case, the sodium ring becomes partial and the flux is weaker than that of Case 1. It is because there are not enough space on the dayside for energetic sodium ions which have large Larmor radii to drift through the dayside magnetosphere. A sample trajectory of sodium ions in the partial sodium ring is shown in Figures $4 \mathrm{~b}$ and $4 \mathrm{~d}$. The trajectory indicates that the partial sodium ring is composed of energetic particles first accelerated in the magnetosheath and then entering into the magnetosphere while drifting along the magnetopause. The entering point of these ions is near the dawnside terminator. Some ions can enter into the magnetosphere from the dayside. However, such ions do not drift around the planet until the nightside, but precipitate onto the planet. Indeed, the dayside magnetosphere is strongly compressed by the solar wind so that the equipotential line of the magnetic field strength intersects the planetary surface on the nightside. The pitch angle of the sodium ion is strongly scattered at the entering point, inducing a large bounce motion while drifting around the planet toward the nightside. 


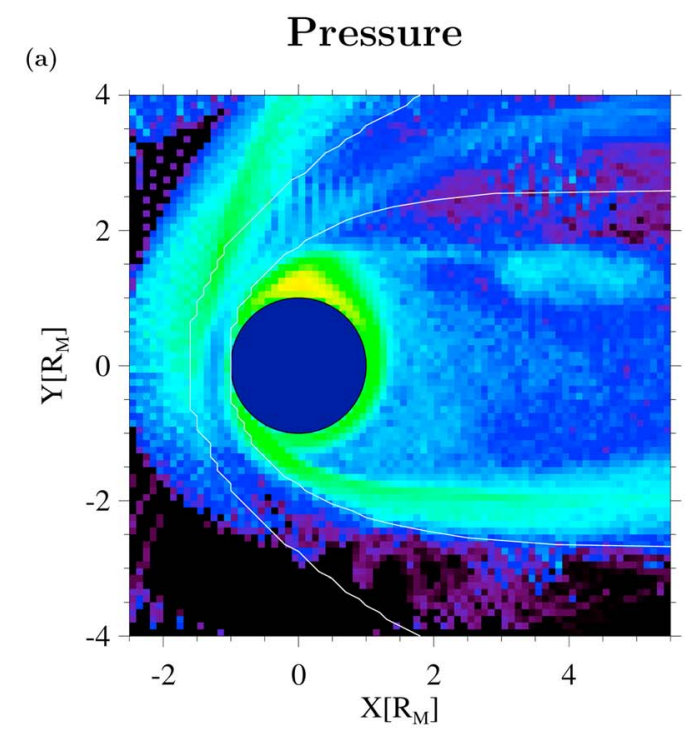

(c)

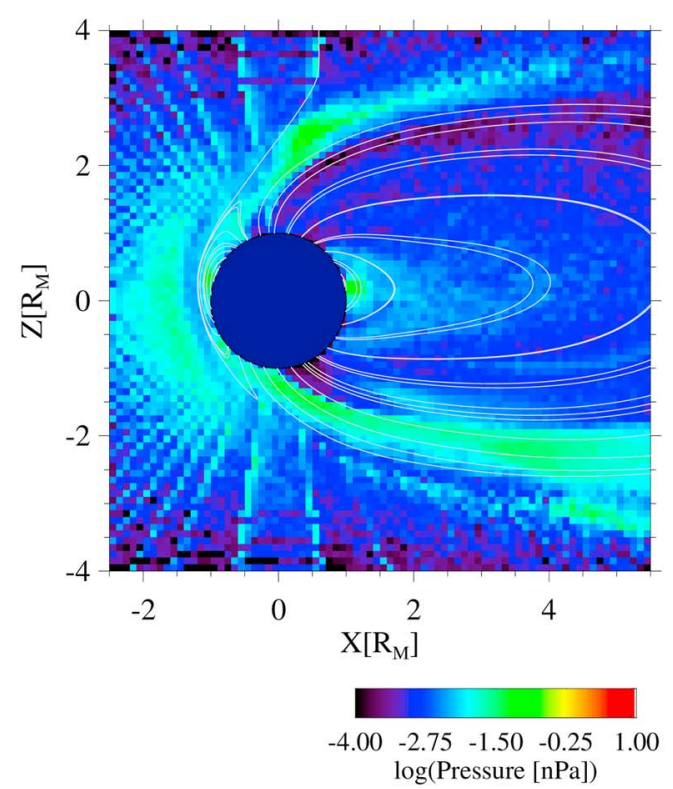

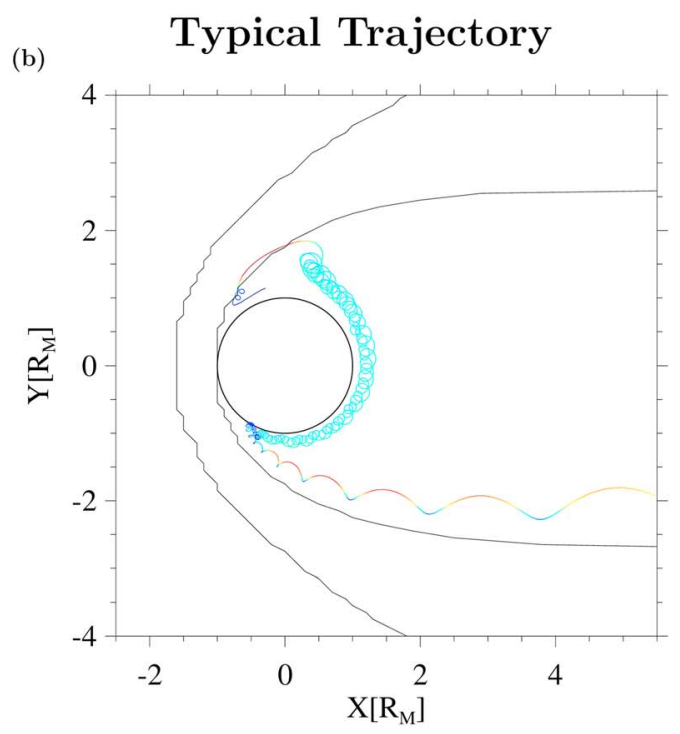

(d)

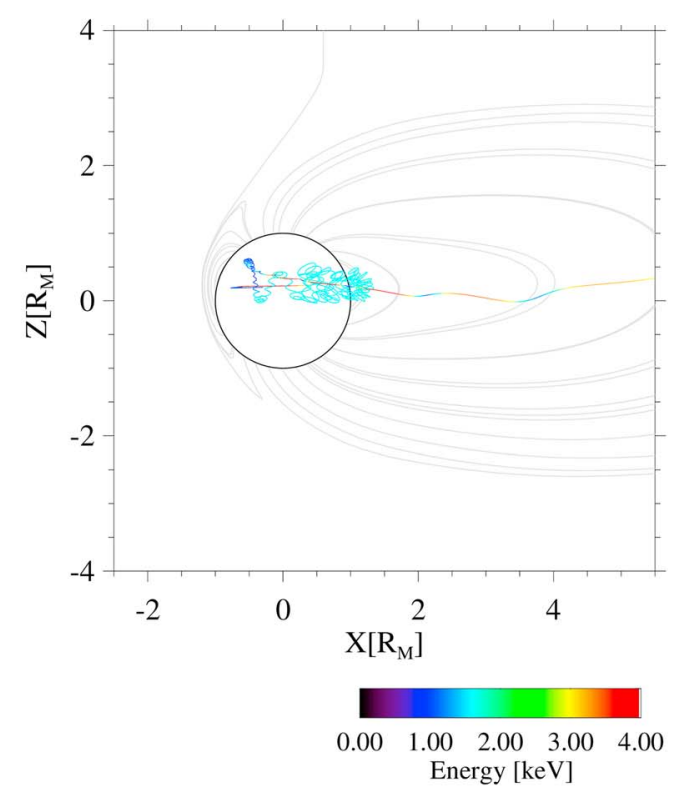

Figure 4. $(a, c)$ The pressure distribution of sodium ions obtained from statistical trajectory tracing in the high solar wind dynamic pressure case (Case 2). (b, d) A sample trajectory of sodium ions in the partial sodium ring (high-pressure region around the planet) is shown. The format of the figure is the same as that in Figure 3.

Figures $5 \mathrm{~b}$ and $5 \mathrm{~d}$ show the distributions of precipitating sodium ions onto the planetary surface for Case 2 . The precipitation region which corresponds to the partial sodium ring consists of a thin but intense band around the magnetic equator in addition to a wide but weak precipitation in the southern hemisphere on the nightside. High latitude precipitation bands of several hundreds of eV also exist with slightly higher energy than in Case 1. In Case 2, cusp precipitation is dominant in the dayside. The southern cusp precipitation is particularly pronounced and widely extended up to the northern cusp region.

\section{Discussions}

\subsection{Structure of Mercury's Magnetosphere}

Without dipole offset, Mercury's magnetosphere becomes symmetric in the northern and southern hemispheres during purely northward IMF conditions (Yagi et al., 2010). One of the outstanding characteristic of 

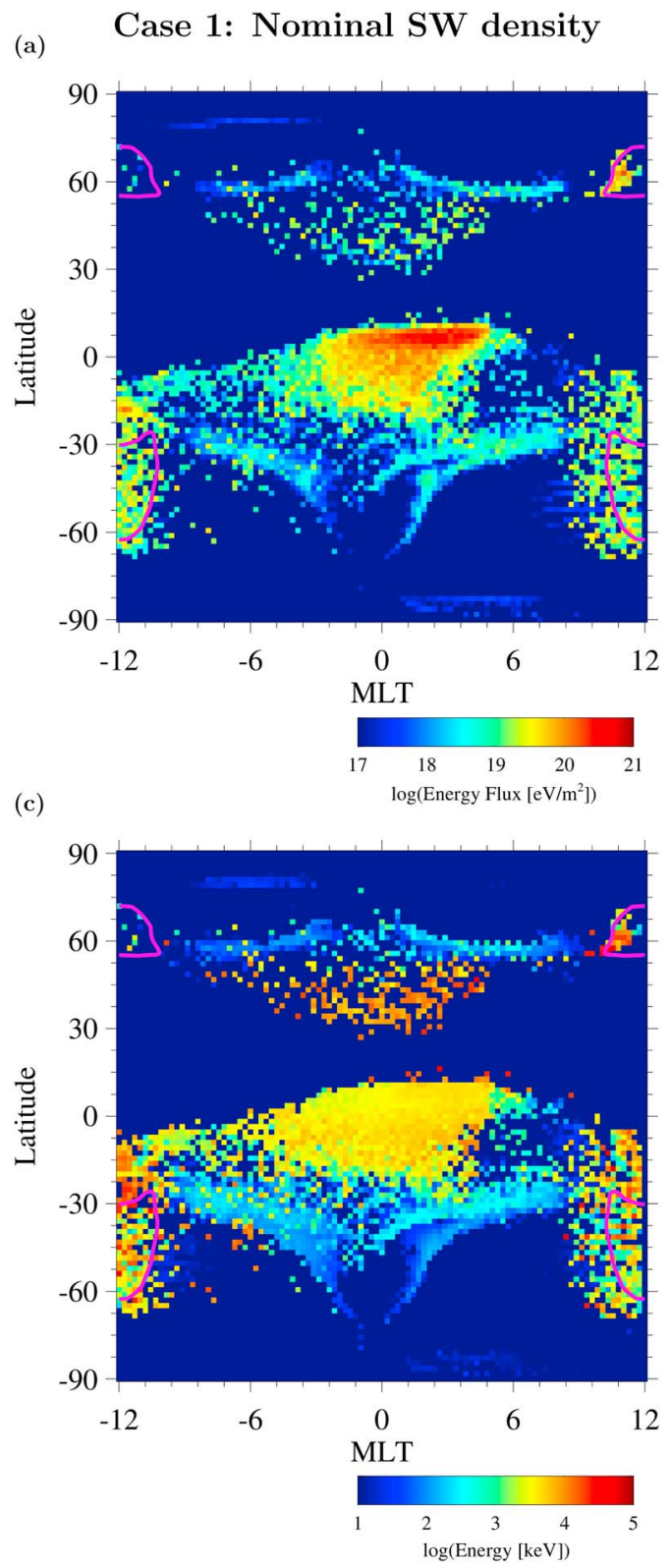
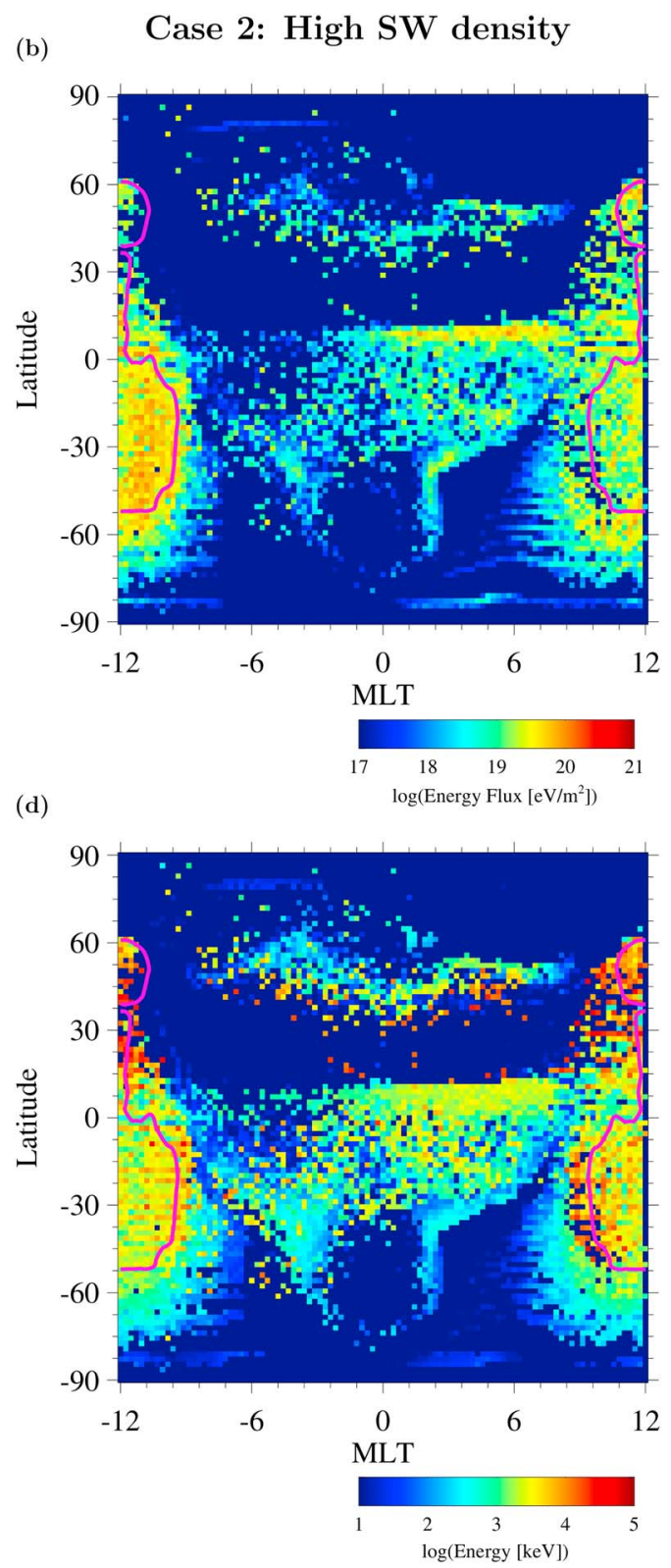

Figure 5. The $(a, b)$ energy flux and $(c, d)$ energy of the precipitating sodium ions onto the planetary surface are shown as a function of latitude and MLT (magnetic local time). Figures 5a and 5c show the typical solar wind case (Case 1), and Figures $5 \mathrm{~b}$ and $5 \mathrm{~d}$ show the high solar wind dynamic pressure case (Case 2 ).

Mercury's magnetosphere is a wide-open field line region around the south pole shown in Figures $1 \mathrm{a}$ and $1 \mathrm{~b}$ for both typical and high solar wind dynamic pressure cases. The asymmetry of the magnetic field structures comes from the asymmetry of the double lobe reconnection (Hasegawa et al., 2006). In our previous study (Yagi et al., 2010), the assumed dipole field strength was $300 \mathrm{nT}$ at equator. A decrease of the dipole field strength to $195 \mathrm{nT}$ based on MESSENGER observations affects the scale of Mercury's magnetosphere, especially the location of magnetopause. Compared to the previous study, the location of the magnetopause moved from 1.7 $R_{M}$ to $1.4 R_{M}$ for typical solar wind conditions (Case 1, Psw = 9.4 nPa), and from $1.4 R_{M}$ to $1.2 R_{M}$ for high solar wind dynamic pressure conditions (Case 2, Psw = $37.4 \mathrm{nPa}$ ). Note that the magnetosphere is so compressed that the magnetopause is difficult to identify in Case 2 . In the $z=0.2$ $R_{M}$ plane, which corresponds to the magnetic equatorial plane of the offset dipole, the tailward flow is dominant in most of the magnetosphere except around the planet for both solar wind cases. There are 
slow return flows around the planet, and this return flow near the dayside magnetopause plays an important role in the formation of the sodium ring, a high sodium pressure region around the planet.

Compared to the observations by MESSENGER, typical magnetopause is located at $1.45 R_{M}$, and it varies from $1.55 R_{M}(P=8.8 \mathrm{nPa})$ to $1.35 R_{M}(P=21.6 \mathrm{nPa})$ by solar wind pressure (Winslow et al., 2013). In both cases, the locations of magnetopause are nearer than the observation, while Case 2 is statistically small. This discrepancy might partly be explained by induction effects which resist the compression of the dayside (Jia et al., 2015). Previous simulation studies also reports that the conductivity of the planet could change the configuration of Mercury's magnetosphere such as plasma sheet boundary, or the strength of magnetospheric convection (Seki et al., 2013). In this study, the planetary surface is set as a "reflective" boundary that is equivalent to "low conductive body" which does not reduce the magnetospheric convection at the vicinity of the planet while the induction effect is not included. It is a simplified model; however, the magnetospheric convection is not so strong because of the northward IMF, and the conductivity of the surface is less effective in this simulation.

\subsection{Dynamics of Sodium lons in Mercury's Magnetosphere}

The previous study indicated that the sodium ring is formed by ions accelerated in the magnetosheath reentering into the magnetosphere. Since the weaker and offset dipole reduces the region of closed drift path, a key question is whether the sodium ring can exist with the offset dipole magnetosphere. As shown in section 3, the sodium ring can exist for typical solar wind conditions (Case 1), while it becomes a partial ring in case of high solar wind dynamic pressure conditions (Case 2).

While drifting around the magnetopause, sodium ion meanders between the magnetosheath and magnetosphere because of their large Larmor radius. Part of these ions are pushed into the magnetosphere by the magnetospheric electric field of convection. To penetrate into Mercury's magnetosphere, the first condition is that the magnetospheric convection is strong enough to overcome or comparable to the magnetic gradient drift across the magnetopause. The magnetospheric convection speed is about $20 \mathrm{~km} / \mathrm{s}$ at maximum, which is equivalent to the $\nabla B$ drift speed of several $\mathrm{keV}$ ions at the magnetopause. This condition determines the upper energy limit of the ions to form a sodium ring. The second condition is that the Larmor radius of sodium ions is larger than the width of the magnetopause in order to be affected by the magnetospheric convection. The width of the magnetopause is about 200 to $300 \mathrm{~km}$, which is equivalent to the Larmor radius of $1 \mathrm{keV}$ sodium ion at the magnetopause. Therefore, the energy range of sodium ring is only of the order of a few keV. This mechanism is valid for both solar wind conditions studied here. It should be noted that this mechanism was only effective for the high solar wind density case in Yagi et al. (2010). Indeed, for nominal solar wind conditions, the subsolar magnetopause was located at $1.7 R_{M}$ and was too far for this mechanism to take place. In this study with a weaker dipole field, the magnetopause is located at 1.4 $R_{M}$ for typical solar wind conditions (Case 1), a location of the magnetopause close enough to lead to the formation of a ring.

Interestingly, bounce motion is induced for sodium ions in the magnetosphere even though sodium ions are picked up at the magnetic equator with pitch angle of $90^{\circ}$. It is because of the north-south asymmetry of the dipole offset in the global MHD simulation. In the northern hemisphere, dayside compression caused by the solar wind could induce the propagation of the magnetic flux toward the magnetotail by the fast compressional mode. In the southern hemisphere, the fast compressional mode is disturbed by the size of the cusp and cannot transport the compressed magnetic flux toward the magnetotail. Therefore, the compression of the dayside magnetosphere is more effective in the southern than in the northern hemisphere. It also creates an asymmetry of the magnetic flux in the magnetotail.

Bounce motions of sodium ions in the sodium ring are amplified on the dayside especially in the southern hemisphere. This type of motion might be explained by drift shell splitting (Walsh et al., 2013), in which ion trajectories bifurcate either northward or southward. Because of the strong dayside compression, two local minima in the magnetic field line are formed at midlatitudes. Because of the north-south asymmetry of magnetic field line, high-energy sodium ions tend to drift southward while drifting in the dayside magnetosphere. Thermal ions are relatively stable in the northern dayside; however, such ions do not contribute to the pressure distribution. In Case 2, the north-south asymmetry of the magnetic field on the dayside is larger than in Case 1; however, no sodium ion can go through the dayside magnetosphere. 
(a)Case 1: Nominal SW density

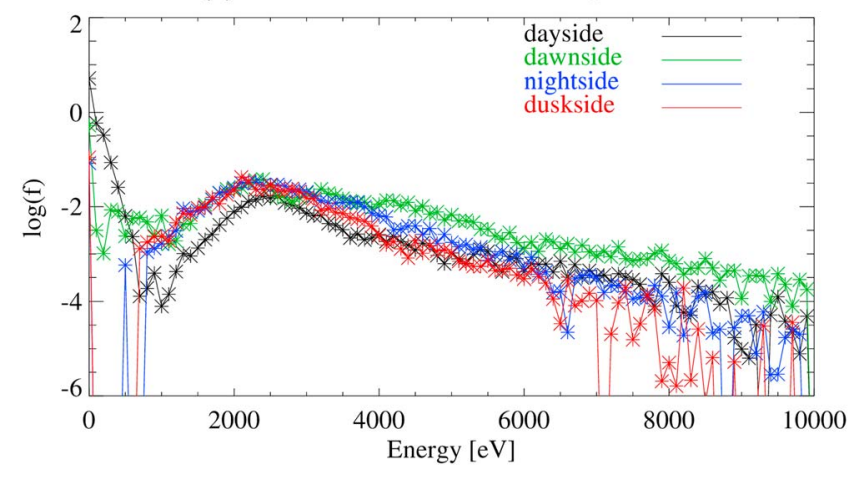

(b)Case 2: High SW density

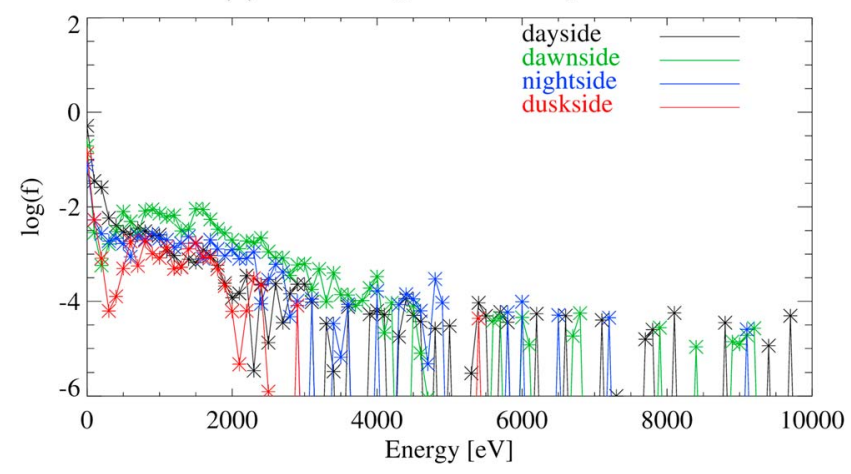

Figure 6. The energy distributions of sodium ions in the dayside (black), dawnside (green), nightside (blue), and duskside (red). (a) The typical solar wind case (Case 1 in the text), (b) the high solar wind dynamic pressure case (Case 2).
Figure 6a shows the energy distributions sampled on the dayside, dawnside, nightside, and duskside in $z=0.2 R_{M}$ plane in Case 1 . Above $1 \mathrm{keV}$, the energy distributions are similar. It indicates that the sodium ring consists of the same ion component simply drifting around the planet by gradient of the magnetic field without changing in energy. The energy distributions have a peak value around $2 \mathrm{keV}$ with power law-like distribution up to $10 \mathrm{keV}$. As for ions with energies higher than $4 \mathrm{keV}$, the dawnside distribution contains larger amount of ions than in the three other regions. It indicates that there is a loss process such as precipitation to the planetary surface while drifting around the planet for the high-energy ions, while lower energy ions can stably drift around the planet from the dawnside to the duskside through nightside. The sodium ring precipitation band shown in Figure $5 \mathrm{a}$ is consistent with this hypothesis. Dayside energy distribution below $4 \mathrm{keV}$ is slightly less dense as in the three other regions. It indicates that there is also some loss process on the dayside for ions with energies less than $4 \mathrm{keV}$.

The energy distributions of sodium ions in Case 2 in the same regions than in Figure 6a are shown in Figure 6b. There is no clear peak in energy. Low-energy ions around $1 \mathrm{keV}$ are dominant, whereas the total ion flux is smaller than that in Case 1. Comparison between the distributions in the dawnside and duskside shows sodium precipitation from several $\mathrm{eV}$ to keV range, which corresponds to the narrow precipitation bands around the magnetic equator from dawnside to nightside. The mechanism of the ions loss is precipitation onto Mercury's surface by finite Larmor radius, as in Case 1. The distortion from an ideal dipole configuration being stronger than in Case 1, the high-energy ions drifting along the equipotential line of magnetic field strength tend to encounter the planet. The energy distribution on the duskside indicates that most ions above $2 \mathrm{keV}$ are lost while drifting from nightside to duskside, while lower energy ions below $2 \mathrm{keV}$ are not.

Notice that these energy ranges of sodium ring depends on the width of magnetopause layer (Yagi et al., 2010), while it is difficult to validate it in the MHD simulation because the width of discontinuities such as magnetopause will be decided by microphysics which is not included in MHD approximation. In addition, $\mathrm{K}-\mathrm{H}$ instability, which is MHD scale physics, could also change the width of layer (while it is not resolved in this study). Therefore, these energy ranges have some uncertainties; however, sodium ring would consist of ions with limited energy range which is related to the width of magnetopause.

The loss processes of sodium ions in the "partial sodium ring" can be classified into three groups. The first process is precipitation into the planet around the magnetic equator because of large Larmor radius. It is also the main process if no dipole offset is considered. The second process is precipitation into the planet by bounce motion, which becomes relatively important in the cases with a dipole offset. The last one is escape to magnetosheath through the duskside magnetopause, because the dayside magnetosphere is thin and most sodium ions cannot go through.

Another loss process, which is related to time variation and is not included in this simulation, might be considered in the real Mercury's magnetosphere. Large amplitude fluctuation of dipole field, which can occur by solar wind variation, for example, destabilizes the trajectories of the ions. The extended periods of the solar wind with steady states are sometimes observed at Earth's orbit (Hasegawa et al., 2006), and the formation of "full sodium ring" could exist only for such occasion. Statistical analysis of sodium ion distribution by MESSENGER also shows a peak at dawnside near Mercury (Raines et al., 2013), which might reflect the entering process at dawnside as discussed in this study. Notice that the statistical analysis integrated the long-term observation including whole solar wind conditions (e.g., northward and southward IMF cases) so that the other peak from nightside to duskside is not shown in this study, but it appears in southward IMF condition (Delcourt et al., 2003). 


\section{Conclusion}

We carried out global MHD simulations of Mercury's magnetosphere under northward IMF conditions using the intrinsic magnetic field model with a dipole offset based on the MESSENGER's observation and statistical trajectory tracing of sodium ions in the electric and magnetic fields obtained from the MHD simulations. The main characteristics of the MHD simulation results are

1. North-south asymmetry especially the open field line region around the southern polar region,

2. Gradual northward deflection of the plasma sheet in the middle and far tail.

The asymmetry of the magnetic fields affects the sodium ion distribution and dynamics. Weaker magnetic field strength in the southern hemisphere than in the north increases ion loss by precipitation into the planetary surface in the southern hemisphere. When the solar wind is nominal, there is enough space for sodium ions to drift on the dayside. In this case, a sodium ring develops that involves high-energy sodium ions drifting around the planet. When the solar wind dynamic pressure is high, the ring becomes partial because the dayside magnetosphere is so compressed that there is no space to drift through but leaks into the duskside magnetosheath. In both cases, the sodium ring is formed by sodium ions that are picked up and accelerated in the magnetosheath just outside the magnetopause and reentered into the magnetosphere due to combination effects of finite Larmor radius and convection electric field on the dawnside magnetosphere. Statistical analysis of sodium ion distribution by MESSENGER show a peak at dawnside near Mercury (Raines et al., 2013), which is consistent with the sodium ring distribution.

\section{Acknowledgments}

M. Yagi thanks N. Terada, Y. Kato, and $\mathrm{H}$. Usui for discussions on Mercury's magnetosphere and encouraging on writing this paper. An authority of the parameters (solar wind and intrinsic magnetic field of Mercury) used in this study are based on the observational data by MESSENGER that are available at Planetary Data System (https://pdsppi.igpp.ucla.edu/).

\section{References}

Anderson, B. J., Slavin, J. A., Korth, H., Boardsen, S. A., Zurbuchen, T. H., Raines, J. M., .. Solomon, S. C. (2011). The dayside magnetospheric boundary layer at Mercury. Planetary and Space Science, 59(15), 2037-2050. https://doi.org/10.1016/j.pss.2011.01.010

Benna, M., Anderson, B. J., Baker, D. N., Boardsen, S. A., Gloeckler, G., Gold, R. E., ... Krimigis, S. M. (2009). Modeling of the magnetosphere of Mercury at the time of the first MESSENGER flyby. Icarus, 209(1), 3-10. https://doi.org/10.1016/j.icarus.2009.11.036

Delcourt, D. C., Grimald, S., Leblanc, F., Berthelier, J.-J., Millilo, A., Mura, A., ... Moore, E. E. (2003). A quantitative model of the planetary Na+ contribution to Mercury's magnetosphere. Annales de Geophysique, 21(8), 1723-1736. https://doi.org/10.5194/angeo-21-1723-2003

Delcourt, D. C., Leblanc, F., Seki, K., Terada, N., Moore, T. E., \& Fok, M.-C. (2007). Ion energization during substorms at Mercury. Planetary and Space Science, 55(11), 1502-1508. https://doi.org/10.1016/j.pss.2006.11.026

Delcourt, D. C., Seki, K., Terada, N., \& Moore, T. E. (2012). Centrifugally stimulated exospheric ion escape at Mercury. Geophysical Research Letters, 39, L22105. https://doi.org/10.1029/2012GL054085

Hasegawa, H., Fujimoto, M., Takagi, K., Saito, Y., Mukai, T., \& Réme, H. (2006). Single-spacecraft detection of rolled-up Kelvin-Helmholtz vortices at the flank magnetopause. Journal of Geophysical Research, 111, A09203. https://doi.org/10.1029/2006JA011728

Hercik, D., Travnicek, P. M., Stverak, A., \& Hellinger, P., (2016), Properties of Hermean plasma belt: Numerical simulations and comparison with MESSENGER data, Journal of Geophysical Research: Space Physics, 121, 413-431. https://doi.org/10.1002/2015JA021938

Ho, G. C., Starr, R. D., Gold, R. E., Krimigis, S. M., Slavin, J. A., Baker, D. N., .. Solomon, S. C. (2011). Observations of suprathermal electrons in Mercury's magnetosphere during the three MESSENGER flybys. Planetary and Space Science, 59(15), 2016-2025. https://doi.org/10.1016/ j.pss.2011.01.011

Ip, W.-H., \& Kopp, A. (2002). Resistive MHD simulations of Ganymede's magnetosphere 2. Birkeland currents and particle energetics. Journal of Geophysical Research, 107(A12), SMP 42-1. https://doi.org/10.1029/2001JA005072

Janhunen, P., \& Kallio, E. (2004). Surface conductivity of Mercury provides current closure and may affect magnetospheric symmetry. Annales de Geophysicae, 22(5), 1829-1837. https://doi.org/10.5194/angeo-22-1829-2004

Jia, X., Slavin, J. A., Gombosi, T. I., Daldorff, L. K. S., Toth, G., \& Holst, B. (2015). Global MHD simulations of Mercury's magnetosphere with coupled planetary interior: Induction effect of the planetary conducting core on the global interaction. Journal of Geophysical Research: Space Physics, 120, 4763-4775. https://doi.org/10.1002/2015JA021143

Kabin, K., Hansen, K. C., Gombosi, T. I., Combi, M. R., Linde, T. J., Dezeeuw, D. L., ... Nagy, A. F. (2000). Global MHD simulations of space plasma environments: heliosphere, comets, magnetospheres of planets and satellites. Astrophysics and Space Science, 274(1/2), 407-421. https:// doi.org/10.1023/A:1026513921198

Kabin, K., Heimpel, M. H., Rankin, R., Aurnou, J. M., Gómez-Pérez, N., Paral, J., ... DeZeeuw, D. L. (2008). Global MHD modeling of Mercury's magnetosphere with applications to the MESSENGER mission and dynamo theory. Icarus, 195(1), 1-15. https://doi.org/10.1016/ j.icarus.2007.11.028

Kallio, E., \& Janhunen, P. (2003). Modelling the solar wind interaction with Mercury by a quasi-neutral hybrid model. Annales de Geophysicae, 21(11), 2133-2145. https://doi.org/10.5194/angeo-21-2133-2003

Kidder, A., Winglee, R. M., \& Harnett, E. M. (2008). Erosion of the dayside magnetosphere at Mercury in association with ion outflows and flux rope generation. Journal of Geophysical Research, 113, A09223. https://doi.org/10.1029/2008JA013038

Korth, H., Anderson, B. J., Johnson, C. L., Winslow, R. M., Slavin, J. A., Purucker, M. E., ... McNutt, R. L. Jr. (2012). Characteristics of the plasma distribution in Mercury's equatorial magnetosphere derived from MESSENGER Magnetometer observations. Journal of Geophysical Research, 117, A00M07. https://doi.org/10.1029/2012JA018052

Leblanc, F., Delcourt, D., \& Johnson, R. E. (2003). Mercury's sodium exosphere: Magnetospheric ion recycling. Journal of Geophysical Research, 108(E12), 5136. https://doi.org/10.1029/2003JE002151

Ness, N. F., Behannon, K. W., Lepping, R. P., \& Whang, Y. C. (1975). The magnetic field of Mercury. I. Journal of Geophysical Research, 80(19), 2708-2716. https://doi.org/10.1029/JA080i019p02708

Ng, K. H., \& Beard, D. B. (1979). Possible displacement of Mercury's dipole. Journal of Geophysical Research, 84(A5), 2115-2117. https://doi.org/ 10.1029/JA084iA05p02115 
Ogata, Y., \& Yabe, T. (1999). Shock capturing with improved numerical viscosity in primitive Euler representation. Computer Physics Communications, 119(2-3), 179-193. https://doi.org/10.1016/S0010-4655(99)00188-5

Pantellini, F., Griton, L., \& Varela, J. (2015). Rarefaction and compressional standing slow mode structures in Mercury's magnetosheath: 3D MHD simulations. Planetary and Space Science, 112, 1-9. https://doi.org/10.1016/j.pss.2015.04.007

Potter, A., \& Morgan, T. (1985). Discovery of sodium in the atmosphere of Mercury. Science, 229, 651-653. https://doi.org/10.1126/ science.229.4714.651

Raines, J. M., Gershman, D. J., Zurbuchen, T. H., Sarantos, M., Slavin, J. A., Gilbert, J. A., ... Solomon, S. C. (2013). Distribution and compositional variations of plasma ions in Mercury's space environment: The first three Mercury years of MESSENGER observations. Journal of Geophysical Research: Space Physics, 118, 1604-1619. https://doi.org/10.1029/2012JA018073

Seki, K., Terada, N., Yagi, M., Delcourt, D. C., Leblanc, F., \& Ogino, T. (2013). Effects of the surface conductivity and the IMF strength on the dynamics of planetary ions in Mercury's magnetosphere. Journal of Geophysical Research: Space Physics, 118, 3233-3242. https://doi.org/ 10.1002/jgra.50181

Simpson, J. A., Eraker, J. H., Lamport, J. E., \& Walpole, P. H. (1974). Electrons and protons accelerated in Mercury's magnetic field. Science, 185(4146), 160-166. https://doi.org/10.1126/science.185.4146.160

Slavin, J. A., Imber, S. M., Boardsen, S. A., DiBraccio, G. A., Sundberg, T., Sarantos, M., ... Solomon, S. C. (2012). MESSENGER observations of a flux-transfer-event shower at Mercury. Journal of Geophysical Research, 117, A00M06. https://doi.org/10.1029/2012JA017926

Travnicek, P., Hellinger, P., \& Schriver, D. (2007). Structure of Mercury's magnetosphere for different pressure of the solar wind: Three dimensional hybrid simulations. Geophysical Research Letters, 34, L05104. https://doi.org/10.1029/2006GL028518

Travnicek, P. M., Schriver, D., Hellinger, P., Hercik, D., Anderson, B. J., Sarantos, M., \& Slavin, J. A. (2010). Mercury's magnetosphere-solar wind interaction for northward and southward interplanetary magnetic field: Hybrid simulation results. Icarus, 209(1), 11-22. https://doi.org/ 10.1016/j.icarus.2010.01.008

Varela, J., Pantellini, F., \& Moncuquet, M. (2015). The effect of interplanetary magnetic field orientation on the solar wind flux impacting Mercury's surface. Planetary and Space Science, 119, 264-269. https://doi.org/10.1016/j.pss.2015.10.004

Walsh, B. M., Ryou, A. S., Sibeck, D. G., \& Alexeev, I. I. (2013). Energetic particle dynamics in Mercury's magnetosphere. Journal of Geophysical Research: Space Physics, 118, 1192-1999. https://doi.org/10.1002/jgra.50266

Whang, Y. C. (1977). Magnetospheric magnetic field of Mercury. Journal of Geophysical Research, 82(7), 1024-1030. https://doi.org/10.1029/ JA082i007p01024

Winslow, R. M., Anderson, B. J., Johnson, C. L., Slavin, J. A., Korth, H., Purucker, M. E., ... Solomon, S. C. (2013). Mercury's magnetopause and bow shock from MESSENGER magnetometer observations. Journal of Geophysical Research: Space Physics, 118, 2213-2227. https://doi.org/ 10.1002/jgra.50237

Xiao, F., Yabe, T., Nizam, G., \& Ito, T. (1996). Constructing a multi-dimensional oscillation preventing scheme for the advection equation by a rational function. Computer Physics Communications, 94(2-3), 103-118. https://doi.org/10.1016/0010-4655(96)00008-2

Yabe, T., \& Aoki, T. (1991). A universal solver for hyperbolic equations by cubic-polynomial interpolation I. One-dimensional solver. Computer Physics Communications, 66(2-3), 219-232. https://doi.org/10.1016/0010-4655(91)90071-R

Yagi, M., Seki, K., Matsumoto, Y., Delcourt, D. C., \& Leblanc, F. (2010). Formation of a sodium ring in Mercury's magnetosphere. Journal of Geophysical Research, 115, A10253. https://doi.org/10.1029/2009JA015226 